\title{
Development of a preliminary diagnostic measure for bovine leukosis in dairy cows using peripheral white blood cell and lymphocyte counts
}

\author{
Masao NISHIIKE ${ }^{1,2}$, Michiyo HAOKA ${ }^{2)}$, Takashi DOI ${ }^{2)}$, Tomoko KOHDA ${ }^{1)}$ and Masafumi MUKAMOTO ${ }^{1) *}$ \\ 1) Department of Veterinary Sciences, Graduate School of Life and Environmental Sciences, Osaka Prefecture University, 1-58 Rinku \\ Orai-Kita, Izumisano, Osaka 599-8531, Japan \\ 2) Livestock Hygiene Service Center, Osaka Prefectural Government, 1-59 Rinku Orai-Kita, Izumisano, Osaka 598-0048, Japan
}

(Received 14 January 2016/Accepted 23 March 2016/Published online in J-STAGE 8 April 2016)

ABSTRACT. Analysis of the association between antibodies against bovine leukemia virus (BLV), BLV proviral load, and white blood cell (WBC) and lymphocyte counts was performed with 774 dairy cows. The average age, WBC counts and lymphoid cell counts tended to be higher in BLV antibody-positive cows than in antibody-negative cows. There was a similar trend in levels of proviral DNA. We analyzed age, WBC counts and lymphocyte counts by principal component analyses to create a distribution chart of the principle component scores. Using the chart, we categorized cows into four quadrants based on additional information, such as the presence of antibody and the levels of proviral DNA. Antibody-positive cows and cows with high BLV proviral load were found mostly in one quadrant of the chart, indicating that it is possible to predict the risk of infection without any knowledge on antibody status by using information, such as WBC counts as a biomarker. When only antibody-positive cows were included in the analysis, a characteristic distribution of different levels of proviral DNA was seen in the quadrants, suggesting that it is possible to estimate the extent of bovine leukosis infection by using this analysis. For this analysis and categorization of the cows into quadrants, we computed a mathematical formulation using discriminant analysis based on age and WBC and lymphocyte counts. This mathematical formulation for the hematological preliminary diagnosis of the disease is recommended as a screening tool to monitor bovine leukosis.

KEY WORDS: biomarker, blood, bovine, diagnosis, leukosis

doi: 10.1292/jvms.16-0022; J. Vet. Med. Sci. 78(7): 1145-1151, 2016

Bovine leukosis can be classified either as an enzootic bovine leukosis (EBL), which is caused by virus infection, or as a sporadic bovine leukosis (SBL), in which viral etiology has not been confirmed. EBL is caused by BLV, which belongs to the genus Deltaretrovirus within the subfamily Orthoretrovirinae and the family Retroviridae. SBL, which is further subdivided into juvenile, thymic and cutaneous $\mathrm{SBL}$, is less common, and therefore, bovine leukosis is most often referred to as an EBL. The first description of bovine leukosis appeared in a German medical journal in 1871, and the disease is therefore thought to have originated in Europe [8]. Characteristic lesions associated with bovine leukosis include systemic lymphoma, which can be diagnosed by palpation of the superficial lymph nodes, and a mass in the pelvic cavity determined by a rectal examination. Not all cattle infected with BLV develop a clinical disease. Around 60 to 70 percent of animals with a BLV infection remain asymptomatic carriers, and approximately 30 percent of infected cattle develop persistent lymphocytosis, a condition considered to be clinically normal. However, after a few months to years of the asymptomatic period, several percent

\footnotetext{
*Correspondence to: Mukamoto, M., Department of Veterinary Sciences, Graduate School of Life and Environmental Sciences, Osaka Prefecture University, 1-58 Rinku Orai-Kita, Izumisano, Osaka 599-8531, Japan. e-mail: mukamoto@vet.osakafu-u.ac.jp (C)2016 The Japanese Society of Veterinary Science

This is an open-access article distributed under the terms of the Creative Commons Attribution Non-Commercial No Derivatives (by-nc-nd) License $<$ http://creativecommons.org/licenses/by-nc-nd/4.0/>.
}

of these infected animals develop B-cell lymphocytic leukemia/lymphoma. In addition to vectors, such as bloodsucking insects, infection routes of bovine leukosis include close contact with infected cattle, for example, during breeding, parturition and milking, as well as the transfer of blood during medical procedures.

With respect to the effect of bovine leukosis on other infectious diseases, there have been reports on an incidence of coinfection with Mycobacterium bovis and BLV in a cow [5] and of impaired responses to $E$. coli bacterin in BLV-positive animals [4], suggesting a possible association between BLV infection and immunosuppression.

A 1996 NAHMS Dairy study conducted in the United States found that $89 \%$ of the U.S. dairy herds were infected with BLV [4]. A study conducted as part of the NAHMS's 1996 survey reported significant economic losses incurred by the U.S. dairy industry from seropositive cows [11]. In order to prevent the spread of this disease, independent investigators from multiple studies report that implementing BLV monitoring programs, for example, by testing for antibodies in serum and milk and by screening for proviral DNA, are important to determine the timeline of infection and to identify seropositive animals $[2,3,6]$.

In addition to these antibody and genetic tests, this disease can also be diagnosed by other methods, such as by measuring lymphocyte counts in the peripheral blood. Based on results from pathologic examination of bovine leukosis, a previous Japanese study demonstrated that careful observation of atypical mononuclear cells by peripheral blood smear is necessary for an accurate diagnosis of aleukemic 
leukemia when the classification is made according to the hematological diagnostic key of the European Community (Key of EC) [10]. In another Japanese study, provisional diagnostic criteria for the preleukemic condition were established based on the classification of persistent lymphocytosis in Japanese Black cattle using a normal lymphocyte count in the peripheral blood and the Bendixen index as references [7]. Another report has confirmed an association between the BLV proviral load and WBC counts in Holstein cows from herds with high BLV seroprevalence, demonstrating that WBC count determination could be a potential tool for monitoring BLV infection levels [1].

The spread of subclinical bovine leukosis can lead to significant economic losses for the dairy cattle industry, and therefore, identifying and culling infected animals has been a major challenge for the industry. In Japan, ELISA antibody tests, as well as real-time PCR tests, are performed on dairy cattle to detect BLV infection. In order to be able to test a large number of dairy cattle, however, it is necessary to reduce the cost of detecting the disease. The aim of this study was to establish diagnostic criteria for bovine leukosis, using hematologic analyses, such as peripheral blood lymphocyte counts, to help develop low-cost testing strategies to screen for BLV infection. In our study, the ELISAs and real-time PCR tests were conducted on milking cows in order to study the associations between BLV infection and WBC and lymphocyte counts, as well as age by multivariate statistical analyses.

\section{MATERIALS AND METHODS}

Study duration and animals: A total of 774 dairy cows from five dairy farms within the prefecture of Osaka, Japan, were studied between 2010 and 2014. The age of these animals was determined by the birth date associated with the individual identification number assigned to each cow.

WBC and lymphocyte counts: Blood samples were collected from tail veins of dairy cows and were directly aliquoted into vacuum blood collection tubes containing EDTA-2Na. Total WBC and lymphocyte counts (cells/ $\mu l$ ) were performed using an automated, veterinary multi-parameter hematology analyzer (Sysmex Corp., Kobe, Japan).

Anti-BLV antibody serum analysis: Blood serum was separated from the blood collected from the tail veins of cattle. Levels of anti-BLV antibodies in the serum were measured according to the manufacturer's instructions by using the BLV-gp51 viral antigen-based anti-BLV antibody ELISA kit (JNC Corp., Tokyo, Japan). The $\mathrm{S} / \mathrm{P}$ ratio is the ratio between the OD value of the sample and the OD value for the positive control provided in the kit. If the $\mathrm{S} / \mathrm{P}$ ratio was equal to or greater than 0.3 , the sample was interpreted as positive. In contrast, if the $\mathrm{S} / \mathrm{P}$ value was lower than 0.3 , the sample was recorded as negative.

Real time polymerase chain reactions (PCR): WBCs were purified from total blood, which was obtained from the tail vein of dairy cows, by red blood cell lysis with ammonium chloride. Total DNA was extracted from the WBCs using a commercially-available DNA isolation kit (DNeasy Blood
\& Tissue Kit: QIAGEN, Hilden, Germany). Real-time PCR for detecting the BLV tax gene was performed with the Cycleave PCR BLV detection kit, a specific probe, primers, a positive control sample for BLV and the Cycleave PCR Reaction Mix SP (Takara Bio Inc., Kusatsu, Japan). We determined the amount of BLV proviral DNA (copies/10 $n \mathrm{~g}$ DNA) on a PCR machine (Thermal Cycler DiceR Real Time System TP800: Takara Bio Inc.). The numerical values of BLV proviral DNA levels were log-transformed. The logtransformed index scores (0: zero or less than zero, 1, 2 and 3) were utilized for the statistical analyses.

Multivariate statistical analysis: The total WBC and lymphocyte counts and ages of all dairy cows examined, as well as the cows that showed BLV seropositvity, were statistically analyzed with a principal component analysis (PCA), a popular multivariate analysis. We calculated both eigenvalues and eigenvectors using the Jacobi's method for accurate archiving [12].

Each principal component was expressed as a linear combination of the standardized variables. The principal component scores were calculated using the eigenvector as a coefficient. The principal component scores, calculated based on the results of the WBC and lymphocyte counts and age of each dairy cow, were plotted in a two-dimensional plane. In order to estimate the extent of BLV infection, the distribution of the principle component scores, with additional information on BLV antibody titer and BLV proviral DNA levels, was plotted, and the data were categorized into quadrants.

In terms of the categorization of the principal component scores into quadrants, a type of multivariate analysis method called discriminant analysis was applied to the data. We developed a mathematical formula for categorizing values into groups using a linear discriminant function based on average vectors and the variance-covariance matrix of the parameters (WBC and lymphocyte counts and age) [12].

Other statistical analysis: In addition to multivariate statistical analyses, we analyzed for statistically significant differences between groups in terms of BLV positivity (Chisquared test) and mean values in each measurement (F-test and Student $t$-test) $[9,13]$.

Ethics statement: All examinations in this study were permitted by the farm owners and conducted as a part of government affairs by veterinarians who were employed as civil servants by prefectural governments and belonged to the Livestock Hygiene Service Center. Any animals were not scarified for the purpose of this study. No human participants were involved in this study. None of the authors have any conflicts of interest. Thus, ethical approval of animal experimentation was not necessary.

\section{RESULTS}

Comparison of the antibody and genetic test results between different age groups and farms: We compared the BLV antibody-positive rates between different age groups in 774 dairy cows up to the age of 12 years. Starting at the age of four years, the incidence of antibody-positive cows began to increase with age, reaching $69 \%$ at the 6- to 8 -year 
Table 1. Comparison of the antibody and genetic test results between different age groups and farms

\begin{tabular}{|c|c|c|c|c|c|c|c|}
\hline \multirow{2}{*}{\multicolumn{2}{|c|}{ Group }} & \multicolumn{4}{|c|}{ Proviral load index values } & \multirow{2}{*}{ No proviral DNA } & \multirow{2}{*}{ Antibody positive rates } \\
\hline & & 3 & 2 & 1 & 0 & & \\
\hline \multirow{5}{*}{$\begin{array}{l}\text { Age } \\
\text { (year) }\end{array}$} & $<2$ & $1 \%$ & $10 \%$ & $1 \%$ & $3 \%$ & $84 \%$ & $16 \%$ \\
\hline & 2 to 4 & $4 \%$ & $18 \%$ & $5 \%$ & $8 \%$ & $65 \%$ & $37 \%$ \\
\hline & 4 to 6 & $9 \%$ & $21 \%$ & $8 \%$ & $15 \%$ & $47 \%$ & $58 \%$ \\
\hline & 6 to 8 & $14 \%$ & $27 \%$ & $11 \%$ & $14 \%$ & $35 \%$ & $69 \%$ \\
\hline & $8 \leq$ & $8 \%$ & $21 \%$ & $9 \%$ & $10 \%$ & $53 \%$ & $54 \%$ \\
\hline \multirow{5}{*}{ Farms } & $\mathrm{A}(90)^{\mathrm{a})}$ & $1 \%$ & $13 \%$ & $1 \%$ & $1 \%$ & $85 \%$ & $15 \%$ \\
\hline & $\mathrm{B}(80)$ & $13 \%$ & $25 \%$ & $8 \%$ & $14 \%$ & $40 \%$ & $64 \%$ \\
\hline & $\mathrm{C}(60)$ & $11 \%$ & $29 \%$ & $10 \%$ & $11 \%$ & $40 \%$ & $67 \%$ \\
\hline & $\mathrm{D}(40)$ & $2 \%$ & $22 \%$ & $15 \%$ & $23 \%$ & $37 \%$ & $69 \%$ \\
\hline & E (40) & $0 \%$ & $3 \%$ & $1 \%$ & $3 \%$ & $93 \%$ & $9 \%$ \\
\hline
\end{tabular}

a) The numerical value indicates breeding number of dairy cows.

Table 2. The comparison of the age and the hematological-finding by the BLV antibody and BLV proviral load

\begin{tabular}{lcccc}
\hline \multicolumn{1}{c}{ Group } & Age & $\begin{array}{c}\text { WBC counts } \\
(\times 100)\end{array}$ & $\begin{array}{c}\text { Lymphocyte counts } \\
(\times 100)\end{array}$ & $\begin{array}{c}\text { Number of dairy } \\
\text { cows examined (heifer) }\end{array}$ \\
\hline Antibody-positive group & $4.9 \pm 2.2^{\mathrm{a}, \mathrm{b})}$ & $110.6 \pm 42.1^{\mathrm{b})}$ & $\left.63.1 \pm 36.8^{\mathrm{b}}\right)$ & $379(14)$ \\
Antibody-negative group & $3.8 \pm 2.6$ & $84.2 \pm 22.3$ & $39.5 \pm 15.7$ & $395(66)$ \\
Proviral load index values & & & & \\
3 & $5.3 \pm 2.1$ & $157.1 \pm 35.3^{\mathrm{d})}$ & $107.4 \pm 29.4^{\mathrm{d})}$ & 56 \\
2 & $4.7 \pm 2.3$ & $123.2 \pm 40.7^{\mathrm{d})}$ & $75.3 \pm 35.0^{\mathrm{d})}$ & 155 \\
1 & $5.1 \pm 2.1$ & $85.2 \pm 22.0$ & $38.4 \pm 13.9$ & 55 \\
0 & $4.8 \pm 2.1$ & $83.2 \pm 22.7$ & $36.9 \pm 11.8$ & 83 \\
No proviral DNA & $3.9 \pm 2.6^{\mathrm{c})}$ & $84.0 \pm 22.1$ & $39.3 \pm 15.6$ & 425 \\
Normal range & & $78.1 \pm 19.4$ & $50.0 \pm 25.0$ & \\
\hline
\end{tabular}

a) average $\pm \mathrm{SD}$. b) This value indicates a statistically significant $(P<0.01)$ difference from the value for antibodynegative group. c) This value indicates a statistically significant $(P<0.01)$ difference from the value for proviral load index values of $0,1,2$ and 3 . d) This value indicates a statistically significant $(P<0.01)$ difference from the value for proviral load index values of 0 and 1 .

age group. Although there was no obvious trend in antibodypositive rates with increasing herd size, the farms in the current study were divided into two categories - herds with greater than $60 \%$ prevalence of antibody-positive cows and herds with low prevalence ( $9 \%$ and $15 \%$ ) (Table 1$)$. We also performed real-time PCR analysis on the same samples and compared BLV proviral load levels between different age groups. The prevalence of BLV-infected animals as well as the proviral load increased with increasing age. However, the proviral load was similar among age groups at 4 years of age or greater (Table 1). The farms were again divided into two categories in terms of the prevalence of BLV-infected animals -herds with high prevalence and herds with low prevalence. With respect to the proviral load, all of the farms in the study had BLV-infected animals with proviral load index values of two or greater (Table 1). When the results of the real-time PCR analysis were compared with those of the antibody test, $7 \%$ of the antibody-positive cows were found to be free of proviral BLV DNA.

When the average values of age, WBC counts and lymphocyte counts were compared between the antibody-positive and negative groups, the average age of the antibody-positive group was found to be higher than that of the negative group. The rate of antibody-positive cases was low in heifers $(18 \% ; 14 / 80)$, suggesting the association between parturition and BLV infection. Both WBC and lymphocyte counts were higher in the antibody-positive group with average WBC counts exceeding the normal range (Table 2).

The cows in our study were classified into five categories according to proviral load levels: cows with no detectable proviral DNA and cows with proviral load index values of $0,1,2$ and 3 . The average values for age, WBC counts and lymphocyte counts were then compared among these groups (Table 2). While there was no significant difference in the age between the four groups with proviral load index values, the average age was significantly lower in the group with no proviral DNA. Cows with proviral load index values of 2 or 3 had significantly higher and above normal WBC and lymphocyte counts than cows with index values of 0 or 1 as well as those with no detectable levels of proviral DNA. There was no significant difference in WBC and lymphocyte counts between groups with low proviral load (i.e. groups with index values of 0 and 1) and the group with no proviral DNA. Both WBC and lymphocyte counts of these cows were within the normal range. WBC and lymphocyte counts of cows with proviral DNA were positively correlated with 


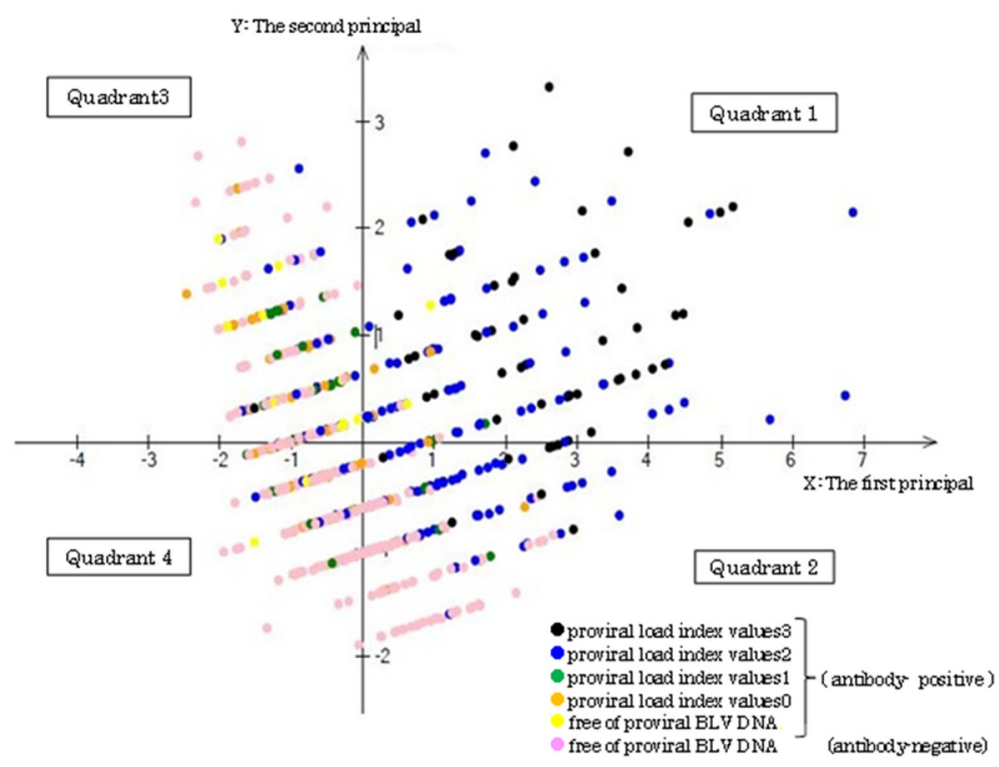

Fig. 1. Combination of principal component analysis (PCA) with anti-BLV antibody titers and BLV proviral DNA levels in each categorized quadrant.

Table 3. Combination of principal component analysis (PCA) with anti-BLV antibody titers and BLV proviral DNA levels in each categorized quadrant.

\begin{tabular}{|c|c|c|c|c|c|c|c|}
\hline \multirow{2}{*}{ Group } & \multirow{2}{*}{$\begin{array}{c}\text { Antibody } \\
\text { positive rates }\end{array}$} & \multicolumn{4}{|c|}{ Proviral load index values } & \multirow{2}{*}{$\begin{array}{l}\text { No proviral } \\
\text { DNA }\end{array}$} & \multirow{2}{*}{$\begin{array}{l}\text { Number of dairy } \\
\text { cows examined }\end{array}$} \\
\hline & & 3 & 2 & 1 & 0 & & \\
\hline Quadrant 1 & $98 \%$ & $38 \%$ & $52 \%$ & $2 \%$ & $3 \%$ & $4 \%$ & 126 \\
\hline Quadrant 2 & $39 \%$ & $4 \%$ & $25 \%$ & $4 \%$ & $5 \%$ & $62 \%$ & 181 \\
\hline Quadrant 3 & $49 \%$ & $0 \%$ & $13 \%$ & $13 \%$ & $15 \%$ & $59 \%$ & 227 \\
\hline Quadrant 4 & $31 \%$ & $0 \%$ & $5 \%$ & $6 \%$ & $15 \%$ & $73 \%$ & 240 \\
\hline
\end{tabular}

proviral load levels, with correlation coefficients of 0.558 and 0.604 , respectively.

Combination of principal component analysis (PCA) with anti-BLV antibody titers and BLV proviral DNA levels in each categorized quadrant: PCA was performed on the numerical values of the age and WBC and lymphocyte counts of 774 dairy cows, using a correlation matrix for each variable. Calculation of the cumulative proportion using the first and second principal components results in a value of 0.974 ; therefore, principal component scores were calculated using the eigenvectors for the first two principal components. The principal component scores were further plotted on a twodimensional (2D) plane, with the first principal component on the $\mathrm{x}$-axis and the second principal component on the $\mathrm{y}$-axis. The $\mathrm{x}$-axis is horizontal and perpendicular to the $\mathrm{y}$ axis. The 2D plot was divided into 4 sections: Quadrant 1 $(x>0, y>0)$, Quadrant $2(x>0, y<0)$, Quadrant $3(x<0, y>0)$ and Quadrant $4(x<0, y<0)$. We investigated the distribution of certain variables (such as the extent of BLV seropositivity and the level of the BLV proviral DNA) within the quadrants of the 2D plot. We found a very high frequency of cattle with a high BLV proviral load index in quadrant 1 (Fig. 1).

The BLV antibody-positive rate of quadrant 1 was $98 \%$ and significantly higher than those of other quadrants; however, the BLV-positive rates of other quadrants were also relatively high, ranging from 30 to $50 \%$ (Table 3 ).

Of those cows in quadrant $1,90 \%$ had proviral load index values of either 2 or 3 . Only $4 \%$ of the cows in quadrant 1 were free of BLV infection. More than half of the cows in other quadrants were found to have no detectable levels of proviral DNA (Table 3).

The average age was high in quadrants 1 and 3 (5.6 and 6.9 years, respectively), whereas the average age was low in quadrants 2 and 4 (1.9 and 3.2 years, respectively) (Table 4).

The average WBC counts of quadrant $1(14,990)$ were the highest of all quadrants, followed by quadrant $2(11,680)$. The WBC counts of both of these quadrants were above normal. There was no statistical difference in the average WBC counts between quadrants $3(7,400)$ and $4(7,600)$, both of which were within normal range (Table 4). The lymphocyte counts showed a similar trend to the WBC counts (Table 4).

PCA of BLV-seropositive cows combined with BLV proviral load in each categorized quadrant: PCA was applied to the numerical age and WBC and lymphocyte counts of 349 dairy cows that showed BLV seropositivity. Since the calculation of the cumulative proportion using the first two 
Table 4. The comparison of the age and the hematological-finding in each categorized quadrant

\begin{tabular}{clccc}
\hline Group & \multicolumn{1}{c}{ Age } & $\begin{array}{c}\text { WBC counts } \\
(\times 100)\end{array}$ & $\begin{array}{c}\text { Lymphocyte counts } \\
(\times 100)\end{array}$ & $\begin{array}{c}\text { Number of dairy } \\
\text { cows examined }\end{array}$ \\
\hline Quadrant 1 & $5.6 \pm 1.8^{\mathrm{a}, \mathrm{b})}$ & $\left.149.9 \pm 36.3^{\mathrm{c}}\right)$ & $\left.100.3 \pm 32.8^{\mathrm{c}}\right)$ & 126 \\
Quadrant 2 & $1.9 \pm 1.1$ & $\left.116.8 \pm 22.8^{\mathrm{c}}\right)$ & $\left.63.7 \pm 18.0^{\mathrm{c}}\right)$ & 181 \\
Quadrant 3 & $6.9 \pm 1.8^{\mathrm{b})}$ & $74.0 \pm 15.6$ & $31.4 \pm 8.9$ & 227 \\
Quadrant 4 & $3.2 \pm 0.9$ & $76.6 \pm 13.7$ & $34.4 \pm 8.2$ & 240 \\
\hline
\end{tabular}

a) average \pm SD. b) This value indicates a statistically significant $(P<0.01)$ difference from the value for Quadrants 2 and 4. c) This value indicates a statistically significant $(P<0.01)$ difference from the value for Quadrants 3 and 4.

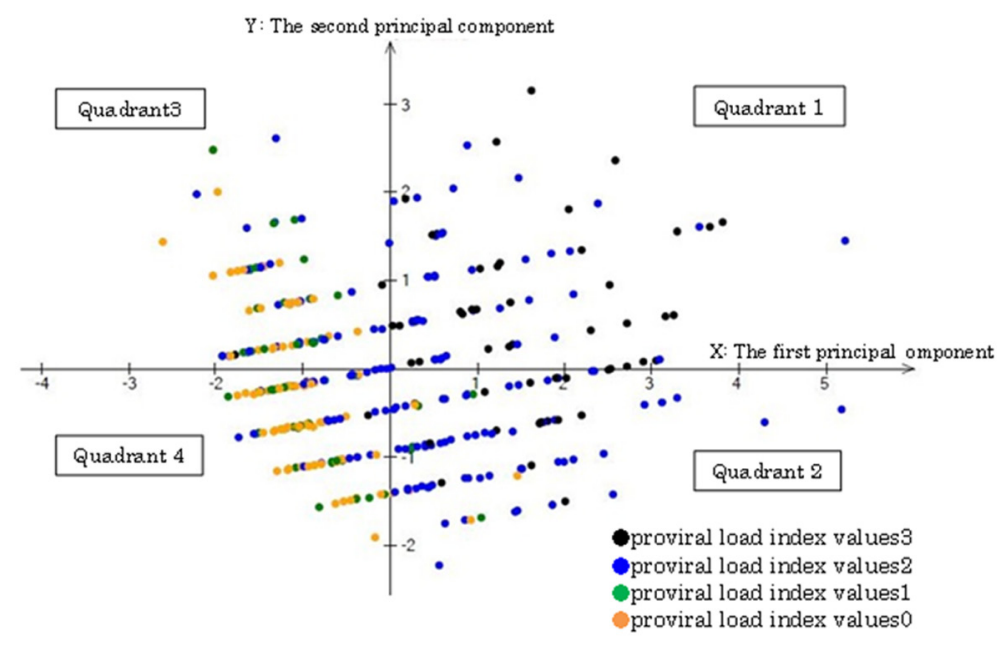

Fig. 2. PCA of BLV-seropositive cows combined with BLV proviral load in each categorized quadrant.

principal components results in a value of 0.981 , the principal component scores were calculated and plotted on a $2 \mathrm{D}$ plot with the first principal component on the $\mathrm{x}$-axis and the second principal component on the y-axis. The $\mathrm{x}$-axis is horizontal and perpendicular to the $y$-axis. The $2 \mathrm{D}$ plot was divided into 4 sections: Quadrant $1(\mathrm{x}>0, \mathrm{y}>0)$; Quadrant 2 $(x>0, y<0)$; Quadrant $3(x<0, y>0)$; and Quadrant $4(x<0$, $y<0)$. We addressed the distribution of the different levels of BLV proviral load within the quadrants of the 2D plot. Cows with high values of the proviral load index were found mostly in quadrants 1 and 2 (Fig. 2). Ninety-nine\% of cows in quadrant 1 were found to have an index value of either $2(50 \%)$ or $3(49 \%)$, and $88 \%$ of cows in quadrant 2 were found to have an index value of $2(69 \%)$ or $3(19 \%)$. On the contrary, $57 \%$ of cows in quadrant 3 and $73 \%$ of cows in quadrant 4 had index values of either 0 or 1; few cows had index values of 3 (4\% in quadrant 3 and $1 \%$ in quadrant 4 ) (Table 5).

The average age was high in quadrants 1 and 3 (6.4 and 7.1 years, respectively), whereas the average age was low in quadrants 2 and 4 (2.7 and 3.9 years, respectively). The average WBC counts of quadrant $1(15,860)$ were the highest of all quadrants, followed by quadrant $2(14,410)$. The WBC counts of both of these quadrants were above normal. There was no statistical difference in the average WBC counts
Table 5. BLV-seropositive cows combined with BLV proviral load in each categorized quadrant

\begin{tabular}{crrrrr}
\hline \multirow{2}{*}{ Group } & \multicolumn{3}{c}{ Proviral load index values } & \multirow{2}{*}{$\begin{array}{c}\text { Number of dairy } \\
\text { cows examined }\end{array}$} \\
\cline { 2 - 5 } & 3 & 2 & 1 & 0 & 74 \\
\hline Quadrant 1 & $49 \%$ & $50 \%$ & $0 \%$ & $1 \%$ & 83 \\
Quadrant 2 & $19 \%$ & $69 \%$ & $7 \%$ & $5 \%$ & 84 \\
Quadrant 3 & $4 \%$ & $39 \%$ & $26 \%$ & $31 \%$ & 108 \\
Quadrant 4 & $1 \%$ & $26 \%$ & $25 \%$ & $48 \%$ & \\
\hline
\end{tabular}

between quadrants $3(8,100)$ and $4(8,300)$, both of which were within normal range. The lymphocyte counts showed a similar trend to the WBC counts (Table 6).

Quantitative classification of BLV infection status with mathematical formulation using discriminant analysis: Discrimination analysis was performed for all dairy cows used in this study, as well as the cows that showed BLV seropositivity. Categorization into the four quadrants of the 2D plot, which was based on principal component scores as well as age and WBC and lymphocyte counts, was analyzed by discriminant analysis. We computed a linear discriminant function in order to distinguish each quadrant, based on age and WBC and lymphocyte counts (Fig. 3). The accuracy of the linear discriminant function for all cattle tested in this 
Table 6. The comparison of the age and the hematological-finding in each categorized quadrant of BLV-seropositive cows

\begin{tabular}{clccc}
\hline Group & Age & $\begin{array}{c}\text { WBC counts } \\
(\times 100)\end{array}$ & $\begin{array}{c}\text { Lymphocyte } \\
\text { counts } \\
(\times 100)\end{array}$ & $\begin{array}{c}\text { Number of dairy } \\
\text { cows examined }\end{array}$ \\
\hline Quadrant 1 & $\left.6.4 \pm 1.6^{\mathrm{a}}, \mathrm{b}\right)$ & $158.6 \pm 33.7^{\mathrm{c})}$ & $108.5 \pm 30.1^{\mathrm{c})}$ & 74 \\
Quadrant 2 & $2.7 \pm 1.0$ & $144.1 \pm 30.3^{\mathrm{c})}$ & $89.8 \pm 27.0^{\mathrm{c})}$ & 83 \\
Quadrant 3 & $7.1 \pm 1.4^{\mathrm{b})}$ & $81.0 \pm 19.7$ & $38.7 \pm 13.8$ & 84 \\
Quadrant 4 & $3.9 \pm 1.0$ & $83.1 \pm 15.5$ & $38.1 \pm 9.9$ & 108 \\
\hline
\end{tabular}

a) average \pm SD. b) This value indicates a statistically significant $(P<0.01)$ difference from the value for Quadrants 2 and 4. c) This value indicates a statistically significant $(P<0.01)$ difference from the value for Quadrants 3 and 4.
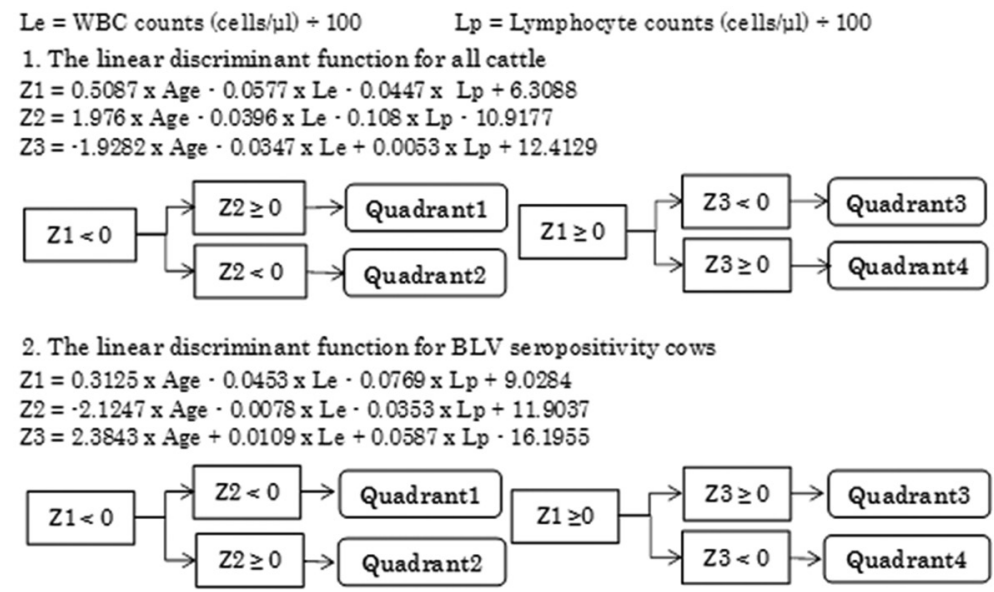

Fig. 3. Quantitative classification of BLV infection status with mathematical formulation using discriminant analysis.

study was calculated as an error of miscategorization for each quadrant. The error rates of Quadrants 1, 2, 3, 4 and overall were $11.9,5.5,13.2,2$ and $7.7 \%$, respectively. With regards to dairy cows with BLV seropositivity, the error rates of Quadrants 1, 2, 3, 4 and overall were 9, 6, 3.5, 0.9 and $4.6 \%$, respectively.

\section{DISCUSSION}

When the BLV antibody-positive rates and the proviral load levels were analyzed by age group, more than half of the animals were found to be infected by the age of 4 years. However, no significant changes in BLV proviral load were observed in cows older than 4 years of age, suggesting that the animals remain persistently infected for an extended period of time. With respect to the hematological findings, the average WBC and lymphocyte counts were higher in antibody-positive cows than those in antibody-negative cows, both of which were above normal. Similarly, the WBC and lymphocyte counts in cows with high values of the proviral load index were above normal, and these counts tended to be higher than those in cows with low or no proviral DNA. Furthermore, levels of proviral DNA were positively correlated with the WBC and lymphocyte counts.
The extent of infection was plotted on a distribution chart which was created using principal component scores calculated from age, WBC counts and lymphocyte counts. In an analysis examining both antibody-positive and negative cows, most of the antibody-positive cows with high proviral load were found in quadrant 1 . BLV-positive cows diagnosed according to the Key of EC were also found mostly in quadrant 1 . However, the distinction between the antibodypositive and negative cows was less clear in the rest of the quadrants, which contained both antibody-positive and negative cows that would have all been classified as normal according to the Key of EC. According to this analysis, since cows grouped into quadrant 1 are older in age and have high WBC and lymphocyte counts, if a cow is categorized into quadrant 1 using these criteria and has not undergone further testing, such as for the presence of BLV antibody, it may be necessary to perform additional serologic testing.

In a similar analysis of antibody-positive cows, we attempted to categorize the extent of infection using the distribution chart created from principal component scores. Among the quadrants into which cows with high WBC and lymphocyte counts were grouped, quadrant 1 which contained older cows was found to have more cows with the highest level of proviral load (index 3), and quadrant 2 
which consisted of younger cows was also found to have more cows with relatively high proviral load (index 2). It was shown by our study that the infection of cows in these quadrants may be progressive and that the cows may have an increased risk of developing the disease. Therefore, if a seropositive cow is categorized into these quadrants, culling or a real-time PCR test is recommended. Although there was a higher number of older cows in quadrant 3 compared to those in quadrant 4 , their proviral load indices were low and the lymphocyte counts were normal in both quadrants. Therefore, their potential risk for developing the disease was low. However, prompt culling of older animals and periodic monitoring of younger animals, for example, by testing for BLV genes, are recommended. When we analyzed the cows according to the Key of EC, BLV-infected animals were mostly found in quadrant 1 , into which most cows with high levels of proviral DNA were categorized in our analysis. On the other hand, many cows classified as clinically normal according to the Key of EC were found in quadrants 3 and 4 , into which cows with low proviral load were categorized. The linear discriminant function for categorizing values into each quadrant achieved an approximate error rate of $10 \%$. These results strongly suggest that this low-error rate linear discriminant function can be a useful tool for monitoring the status of BLV infection in dairy cows using blood cell counts, such as WBC counts.

In summary, our analysis makes it possible to predict the proviral load levels in cows for which the pathological condition is difficult to assess using the Key of EC. Our method has proven to be useful as a hematological basis for culling cows with BLV infections. Blood sampling is an essential procedure for screening not only bovine leukosis but also other diseases; therefore, our method can be used as a lowcost screening procedure for bovine leukosis in tandem with other periodic testing used for various diseases. It will help contribute to setting up a management plan for dairy farms that try to seek preventive methods against infection, such as monitoring the route of viral transmission and culling BLVinfected cows.

In the future, establishing a management system to collect data, such as WBC counts, on cows that have definitively developed the disease, as well as diligently analyzing the data, will be necessary to improve the predictive accuracy of risk assessment for disease development.

\section{REFERENCES}

1. Alvarez, I., Gutiérrez, G., Gammella, M., Martínez, C., Politzki, R., González, C., Caviglia, L., Carignano, H., Fondevila, N., Poli, M. and Trono, K. 2013. Evaluation of total white blood cell count as a marker for proviral load of bovine leukemia virus in dairy cattle from herds with a high seroprevalence of antibodies against bovine leukemia virus. Am. J. Vet. Res. 74: 744-749. [Medline] [CrossRef]

2. Bartlett, P. C., Norby, B., Byrem, T. M., Parmelee, A., Ledergerber, J. T. and Erskine, R. J. 2013. Bovine leukemia virus and cow longevity in Michigan dairy herds. J. Dairy Sci. 96: 1591-1597. [Medline] [CrossRef]

3. Bartlett, P. C., Sordillo, L. M., Byrem, T. M., Norby, B., Grooms, D. L., Swenson, C. L., Zalucha, J. and Erskine, R. J. 2014. Options for the control of bovine leukemia virus in dairy cattle. $J$. Am. Vet. Med. Assoc. 244: 914-922. [Medline] [CrossRef]

4. Erskine, R. J., Bartlett, P. C., Sabo, K. M. and Sordillo, L. M. 2011. Bovine Leukemia Virus Infection in Dairy Cattle: Effect on Serological Response to Immunization against J5 Escherichia coli Bacterin. Vet. Med. Int. 2011: 915747. [Medline] [CrossRef]

5. Fitzgerald, S. D., Sledge, D. G., Maes, R., Wise, A. and Kiupel, M. 2009. Coinfection of a cow with Bovine leukemia virus and Mycobacterium bovis. J. Vet. Diagn. Invest. 21: 878-882. [Medline] [CrossRef]

6. Gutiérrez, G., Alvarez, I., Politzki, R., Lomónaco, M., Dus Santos, M. J., Rondelli, F., Fondevila, N. and Trono, K. 2011. Natural progression of Bovine Leukemia Virus infection in Argentinean dairy cattle. Vet. Microbiol. 151: 255-263. [Medline] [CrossRef]

7. Ishihara, K., Onuma, M. and Ohtani, T. 1979. Clinical studies on leukemia in Japanese Black cattle. I. Peripheral lymphocyte counts of normal Japanese Black cattle and the hematological diagnostic criteria to establish their preleukemic condition. Jpn. J. Vet. Sci. 41: 103-108. [Medline] [CrossRef]

8. Johnson, R. and Kaneene, J. B. 1992. Bovine Leukaemia Virus and Enzootic Bovine Leukosis. Vet. Bull. 62: 287-312.

9. Kariya, T. 1979. The statistics for medicine and biology. Kyoritu Shuppan Co., Tokyo.

10. Ohshima, K., Ozai, Y., Okada, K. and Numakunai, S. 1980. Pathological studies on aleukemic case of bovine leukosis. Jpn. J. Vet. Sci. 42: 297-309. [Medline] [CrossRef]

11. Ott, S. L., Johnson, R. and Wells, S. J. 2003. Association between bovine-leukosis virus seroprevalence and herd-level productivity on US dairy farms. Prev. Vet. Med. 61: 249-262. [Medline] [CrossRef]

12. Tanaka, Y. and Wakimoto, K. 1983. Methods of Multivariate Statistical Analysis. Gendai-Sugakusha, Kyoto.

13. Yoshida, M. and Abe, T. 1984. The statistical method about the stock raising. Japan Livestock Industry Association, Tokyo. 